\title{
Reconstruction of cosmic history from a simple parametrization of $H$
}

\author{
S. K. J. Pacif ${ }^{1}$, R. Myrzakulov ${ }^{2}$, S. Myrzakul ${ }^{3}$ \\ ${ }^{1}$ Centre for Theoretical Physics, \\ Jamia Millia Islamia, \\ New Delhi 110025, India \\ ${ }^{2}$ Eurasian International Center for Theoretical Physics, \\ Eurasian National University, Astana 010008, Kazakhstan \\ ${ }^{3}$ Department of Theoretical and Nuclear Physics, \\ Al-Farabi Kazakh National University, Al-Farabi av. 71, 050040, \\ Almaty, Kazakhstan \\ shibesh.math@gmail.com ${ }^{1}$,rmyrzakulov@gmail.com², \\ shynaray1981@gmail.com ${ }^{3}$
}

October 1, 2018

\begin{abstract}
In this paper, we propose a simple parametrization of the Hubble parameter $H$ in order to explain the late time cosmic acceleration. We show that our proposal covers many models obtained in different schemes of parametrization under one umbrella. We demonstrate that a simple modification in the functional form of Hubble parameter can give rise to interesting cosmological phenomena such as big rip singularity, bounce and others. We have also constrained the model parameters using the latest 28 points of $H(z)$ data for three cases which admit transition from deceleration to acceleration.
\end{abstract}

Keywords: Hubble parameter, parametrization, acceleration, dark energy

\section{Introduction}

One of the aims of cosmology is to determine a mathematical model of the large scale structure of the Universe which can explain the results of astronomical observations and whose dynamics can be determined by the physical laws describing the behavior of matter on larger scale. According to Alan Sandage [1, in cosmology at the background level, one search for two numbers: $H_{0}$ and $q_{0}$ (suffix ' 0 ' stands for the present value of the quantity), where $H$ (Hubble parameter (HP)) and $q$ (deceleration parameter(DP)) are two dynamical quantities which tell 
about the expansion rate of the Universe. But the present day cosmology use around four to twenty parameters to explain the Universe. Still, $H$ and $q$ play the central role in the Einstein's field equations (EFEs) explaining the observations. They can be defined naturally in the linear and non linear derivatives of scale factor $a(t)$ in the Taylor series expansion of $a(t)$ in the vicinity of the present time $t_{0}$ as

$$
a(t)=a\left(t_{0}\right)+\dot{a}\left(t_{0}\right)\left[t-t_{0}\right]+\frac{1}{2} \ddot{a}\left(t_{0}\right)\left[t-t_{0}\right]^{2}+\cdots .
$$

An overhead dot '.' represents derivative w.r.t. cosmic time ' $t$ '. From equation (11), we obtain

$$
\frac{a(t)}{a\left(t_{0}\right)}=1+H_{0}\left[t-t_{0}\right]-\frac{q_{0}}{2} H_{0}^{2}\left[t-t_{0}\right]^{2}+\cdots,
$$

where

$$
H(t)=\frac{\dot{a}}{a}, q(t)=-\frac{a \ddot{a}}{\dot{a}^{2}}
$$

Till today the most successful theory explaining the Universe is the big bang theory which is based on general relativity. After Hubble's work, cosmologists made attempts to measure the deceleration of the expansion with the belief that the expansion of the Universe must slow down caused by gravity. However, the observations of distant supernovae of type Ia by Supernova Cosmology Project [2] and the High-Z Supernova Search team [3] gave totally unexpected result to the fact that the expansion of the Universe is accelerating. Since then further searches presented convincing evidence for accelerating expansion with greater accuracy [4], [5], [6], [7], [8], [9]. The fact is also supported by some other observations such as CMB [10], [11], BAO [12], [13], SDSS [14, [15] etc. For both cosmology and physics, the cosmic acceleration is probably an important discovery. It raised a lot of questions on the fundamental principles funded with cosmology. Based on the accelerating expansion of the Universe, the past few years produced a plethora of cosmological models either by modifying the energy momentum tensor in the right hand side of Einstein's field equation (EFE) or by modifying the gravity theory (modifying the LHS of EFE). Alternative theories are also there such as the inclusion of inhomogeneity, back reaction, averaging etc. Recently, a series of papers by Vishwakarma [16], [17], [18], [19] explained this fact in a simple and viable way which raised some questions on the geometrization of gravity theory.

Although there are several ways to describe this cosmic acceleration, but it is generally attributed to the presence of dark energy (DE) throughout the Universe. Obviously it gives rise to the question of what this mysterious DE really is, what is its nature and why it starts dominating the Universe so recently. The literature contains numerous models of DE but the simplest and popular candidate of DE is the Einstein's cosmological constant $(\Lambda)$ [20], [21], [22]. However, it suffers from the well known cosmological constant problem [23] which can be alleviated by considering a dynamically decaying $\Lambda$. On the other hand primordial inflation has taken a special status in explaining the origin of the anisotropies in the CMB radiation and the formation of large scale structures. This motivates theorists to invoke scalar field to explain the early and the late time acceleration together. So far, a wide variety of scalar field models 
of $\mathrm{DE}$ have been proposed in the past few years including quintessence [24], 25], [26], 27], K-essence [28], [29], spintessence [30], tachyon [31], [32], quintom [33], [34], [35], [36], chameleon [37], 38], [39] and many more. Though these scalar field models give the equation of state

(EoS) parameter $\left(w=\frac{p}{\rho}\right),-\frac{1}{3}<w<0$, observational data also allow models of DE with EoS parameter crossing -1 line (called phantom field models). A number of phantom field models have been proposed [40, 44], 42, 443, 44, 45] in the past few years. Another way to explain the acceleration is to incorporate the Chaplygin gas [46], [47] into the EFEs. For a brief review on candidates of $\mathrm{DE}$ and alternatives to $\mathrm{DE}$, one can see [48, [49], [50], [51], [52], [53, [54].

Observations suggest that the cosmic acceleration is a recent phenomena and the Universe has entered a state of accelerating expansion around redshift $z \sim 0.5$. The existence of decelerated expansion phase in the Universe is also supported by the gravitational instability theory of structure formation and of big bang nucleosynthesis. This implies that the Universe must have undergone from decelerated to accelerated phase of evolution. This motivates the theorists in modeling the Universe with deceleration to acceleration phase transition. The kinematic approach is discussed in [55] to explain the cosmic acceleration which do not assume the validity of general relativity or any particular gravitational theory (see [56]). This method do not effect the physical or geometrical properties of DE and is known as the model-independent way to study the DE i.e. by a parametrized EoS of dark energy. For a review on parametrization of equation of state parameter $w$, one can see [57. Another model-independent way to study the $\mathrm{DE}$ is by parametrizing the DP. For a brief review on DP, one can see [58], [59]. Also, there are several parametrization of HP considered by Nojiri and Odintsov (and collaborators) 60, to study the future cosmological singularities. Here, in this paper, we have studied the evolution of the Universe by parametrizing the functional form of $H$ and see how it reduces to some known obtained models and explains the late time cosmic acceleration.

\section{Motivation}

We would like to stress on the cosmographic parameter $H$ describing the expansion of the Universe and its role in generating some interesting mathematical models of the Universe in Einstein's theory of gravitation. In FRW cosmology, there are three variables namely $a(t), \rho(t)$, $p(t)$ with two independent equations which can be solved by supplementing the equation of state(s), $w=\frac{p}{\rho}$ of the energy component(s). In this case the parameter $w$ is a constant. For a dynamical $\Lambda$, one needs one more constrain equation to close the system. This extra constrain equation (or the supplementary equation) has been chosen in various ways in order to explain the standard cosmological problems such as to solve the cosmological constant problem, singularity problem, horizon problem, flatness problem, density fluctuation problem, dark matter problem, exotic relics problem, thermal state problem etc. during the past forty-fifty years. With the addition of the DE component into the field equations the EoS parameter $w$ becomes dynamical $(w(t))$. Thus, there are many traditional ways to choose this supplementary equation relating any two variables involved in the field equations e.g. $p \sim \rho, \Lambda \sim a^{-2}, \Lambda \sim \rho$ etc. Also, one can parametrize any variable to get this extra constrain equation to close the system. The various parametrization used in literature relating to $a(t), \rho(t), p(t), \Lambda(t), q(t)$ or $w(t)$ are summarized here in detail (see table-6 to table-11 in appendix-1). 
Also there are various parametrization of Hubble parameter $H(t)$ in literature used to explain some problems of standard cosmology and are listed in the below table- 1 .

Table-1

\begin{tabular}{|l|c|}
\hline Parametrization of $\mathrm{HP}(H)$ & Ref. \\
\hline$H(a)=D a^{-m}(D$ and $m$ are constants $)$ & {$[61]$} \\
\hline$H(a)=e^{\frac{1-\gamma a^{2}}{\alpha a}}(\gamma$ and $\alpha$ are constants $)$ & {$[62]$} \\
\hline$H(a)=\alpha\left(1+a^{-n}\right)(\alpha$ and $n$ are constants $)$ & {$[63]$} \\
\hline$H(t)=\frac{m}{\alpha t+\beta}(\alpha, \beta, m$ are constants $)$ & {$[64],[65]$} \\
\hline$H(t)=\frac{\alpha t_{R}}{t\left(t_{R}-t\right)}\left(\alpha\right.$ is a constant, $t_{R}$ is big Rip time $)$ & {$[66]$} \\
\hline$H(t)=\frac{\alpha}{3}\left(t+T_{0}\right)^{3}-\beta\left(t+T_{0}\right)+\gamma$, & {$[67],[39]$} \\
$\gamma=-\frac{\alpha}{3} T_{0}^{3}+\beta T_{0}\left(\alpha, \beta, T_{0}\right.$ are constants $)$ & \\
\hline$H(t)=H_{0} e^{\lambda t}\left(H_{0}, \lambda\right.$ are constants $)$ & {$[68]$} \\
\hline$H(t)=c_{0}+b_{0}\left(t_{s}-t\right)^{\alpha}\left(c_{0}, b_{0}, \alpha\right.$ are constants $)$ & {$[69]$} \\
\hline$H(t)=H_{0}-H_{1} e^{-\beta t}\left(H_{0}>0, H_{1}>0, \beta\right.$ are constants $)$ & {$[70]$} \\
\hline$H(t)=f_{1}(t)+f_{2}(t)\left(t_{s}-t\right)^{\alpha}$ & {$[60]$} \\
$\left(f_{1}(t) \& f_{2}(t)\right.$ are arbitrary functions, $\alpha$ constant $)$ & \\
\hline
\end{tabular}

One can find some more parametrization of HP in [60].

From equation (3), we find

$$
\begin{gathered}
a(t)=C e^{\int H(t) d t} \text {, where } C \text { is a constant of integration. } \\
\qquad q(t)=-1+\frac{d}{d t}\left(\frac{1}{H(t)}\right)
\end{gathered}
$$

EFEs can also be expressed as

$$
\begin{gathered}
\sum \rho_{i}(t)=3 M_{P l}^{2}\left[(H(t))^{2}+\frac{k}{a^{2}}\right] \\
\sum\left[1+3 w_{i}(t)\right] \rho_{i}(t)=6 M_{P l}^{2}(H(t))^{2}\left[-1+\frac{d}{d t}\left(\frac{1}{H(t)}\right)\right] .
\end{gathered}
$$

In the above equations all the physical variables are in terms of $H(t)$. Now, it is easy to see that a simple integrable form of $H(t)$ will determine all the physical variables smoothly. We prefer to parametrize the HP because the variation of Hubble's law assumed is not inconsistent with observations and has the advantage of providing simple functional form of the time evolution of the scale factor and so as dynamics. Motivated by the above discussions, we propose a simple and convenient form of HP as an explicit function of cosmic time ' $t$ ' in the form

$$
H(t)=\frac{\beta t^{m}}{\left(t^{n}+\alpha\right)^{p}}
$$

where $\alpha, \beta \neq 0, m, n, p$ are real constants (better call them model parameters). $\alpha$ and $\beta$ both have the dimensions of time. The specific values of $m, n, p$ will suggest the different forms of $\mathrm{HP}$ and produce interesting cosmologies. Our parametrization generalizes several known models 
which were obtained by the parametrization of any cosmological parameters $a(t), H(t), q(t)$, $\Lambda(t), \rho(t)$ or $w(t)$ in different contexts. In the next section, we formulate the Einstein's field equations for a general scalar field cosmology and solve the system with the help of our main ansatz.

\section{$3 \quad$ Field equations and solutions}

We know scalar fields are extremely important in modern physics being invariant under coordinate transformations. There have been a great activity in modelling the Universe with a motivation to explain both the early and late time acceleration of the Universe with scalar fields. We know, the nature of DE remain matters of speculation, but it is generally believed to be homogeneous, not very dense and is not known to interact through any of the fundamental forces other than gravity. So, it can be represented as large scale scalar field $\phi$. For an ordinary scalar field $\phi$ minimally coupled to gravity with Lagrangian density $\mathcal{L}=-\frac{1}{2} g^{\mu \nu} \partial_{\mu} \phi \partial_{\nu} \phi-V(\phi)$, the action is given by

$$
S=\int d^{4} x \sqrt{-g}\left[-\frac{1}{2} g^{\mu \nu} \partial_{\mu} \phi \partial_{\nu} \phi-V(\phi)\right]
$$

where $V(\phi)$ is the potential of the field. The stress-energy tensor of the field $\phi$ take the form of a perfect fluid as [21]

$$
T_{\mu \nu}^{\phi}=\left(\rho_{\phi}+p_{\phi}\right) U_{\mu} U_{\nu}+p_{\phi} g_{\mu \nu}
$$

where the density and pressure of scalar field are expressed as $\rho_{\phi}=\frac{\dot{\phi}^{2}}{2}+V(\phi)$ and $p_{\phi}=$ $\frac{\dot{\phi}^{2}}{2}-V(\phi)$, with the understanding that $\phi$ is spatially homogeneous. The evolution of the scalar field is governed by the wave equation $\ddot{\phi}+3 H \dot{\phi}+V^{\prime}(\phi)=0$, where a prime denotes differentiation with respect to $\phi$. The state equation of scalar field $w_{\phi}$ can be represented as $w_{\phi}=\frac{p_{\phi}}{\rho_{\phi}}=\frac{-1+\frac{\dot{\phi}^{2}}{2 V}}{1+\frac{\phi^{2}}{2 V}}$. This give rise to several candidates for DE, which depends upon the dynamics of the field $\phi$ and its potential energy $V(\phi)$. For a slow roll scalar field $\frac{\dot{\phi}^{2}}{2} \ll V(\phi)$, it reduces to the case of most favoured cosmological constant $\Lambda$ for which $w_{\phi}=-1$. For $-\frac{1}{3}<w_{\phi}<0$ we have quintessence and $w_{\phi}$ crossing -1 , phantom field is observed. To introduce DE into EFEs, we replace the energy momentum tensor $T_{\mu \nu}$ by

$$
T_{\mu \nu}^{\text {Total }}=T_{\mu \nu}+T_{\mu \nu}^{\phi}=\left(\rho_{\text {Total }}+p_{\text {Total }}\right) U_{\mu} U_{\nu}+p_{\text {Total }} g_{\mu \nu}
$$

with the understanding that $\rho_{\text {Total }}=\rho_{\text {eff }}=\rho+\rho_{\phi}$ and $p_{\text {Total }}=p_{\text {eff }}=p+p_{\phi}$.

With the fluid described here by (11), the EFEs reduce to

$$
\begin{gathered}
\rho_{e f f}=\rho+\rho_{\phi}=3 M_{P l}^{2}\left(H^{2}+\frac{k}{a^{2}}\right), \\
p_{e f f}=p+p_{\phi}=-M_{P l}^{2}\left(2 \frac{\ddot{a}}{a}+H^{2}+\frac{k}{a^{2}}\right),
\end{gathered}
$$

with the state equations 


$$
p=w \rho \quad(0 \leqslant w \leqslant 1) \quad \text { and } \quad p_{\phi}=w_{\phi} \rho_{\phi} .
$$

Meanwhile we consider the minimal interaction between matter and dark energy which yield

$$
\dot{\rho}+3 H(1+w) \rho=0, \quad \dot{\rho}_{\phi}+3 H\left(1+w_{\phi}\right) \rho_{\phi}=0,
$$

leading to $\rho \sim a^{-3(1+w)}$ and $\rho_{\phi} \sim a^{-3\left(1+w_{\phi}\right)}$ (for constant $w_{\phi}$ (such as cosmological constant)). But, $w_{\phi}$ must be a function of time in general.

With our main ansatz (8) the general expressions for the time variation of all the $\mathrm{CP}$ are obtained as follows

$$
\begin{gathered}
a(t)=C e^{\beta \int \frac{t^{m} d t}{\left(t^{n}+\alpha\right)^{p}}, C \text { is an arbitrary constant of integration, }} \\
q(t)=-1+\frac{1}{\beta}\left\{(n p-m) t^{n}-m \alpha\right\} \frac{\left(t^{n}+\alpha\right)^{p-1}}{t^{m+1}} \\
\rho_{e f f}(t)=3 M_{P l}^{2}\left[\frac{\beta^{2} t^{2 m}}{\left(t^{n}+\alpha\right)^{2 p}}+\frac{k}{C^{2}} e^{\left.-2 \beta \int \frac{t^{m} d t}{\left(t^{n}+\alpha\right)^{p}}\right]}\right. \\
p_{e f f}(t)=M_{P l}^{2}\left[\left(-3+\frac{2}{\beta}\left\{(n p-m) t^{n}-m \alpha\right\} \frac{\left(t^{n}+\alpha\right)^{p-1}}{t^{m+1}}\right) \frac{\beta^{2} t^{2 m}}{\left(t^{n}+\alpha\right)^{2 p}}-\frac{k}{C^{2}} e^{-2 \beta \int \frac{t^{m} d t}{\left(t^{n}+\alpha\right)^{p}}}\right] \\
\rho(t)=\left[D C^{-3(1+w)}\right] e^{-3(1+w) \beta \int \frac{t^{m} d t}{\left(t^{n}+\alpha\right)^{p}}, D \text { is an arbitrary constant of integration. }}
\end{gathered}
$$

We observe that some particular values of $m, n, p$ will give explicit solutions of EFEs. In general there are one, two or three model parameters in all the parametrization considered (see table- 1 and table- 6 to table-11). But, we have five parameters $\alpha, \beta, m, n, p$ in the functional form of HP. Without the loss of generality, we reduce the number of model parameters by giving some specific values to $m, n, p$ which will be helpful to analyze the physical and geometrical behavior of our obtained models. For some suitable choice of integral values of $m, n, p$ (and one non-integral value of $p$ ), we obtain some specific models leaving $\alpha$ and $\beta$ generic. The various models thus obtained with two model parameters $\alpha$ and $\beta$ are given in the following table- 8 . The two model parameters $\alpha$ and $\beta$ can be constrained from any observational data (e.g. Sne Ia data, $\mathrm{H}(\mathrm{z})$ data or $\mathrm{BAO}$ data). However, one can also constrain all five model parameters simultaneously but in this work we confined to two model parameters $\alpha, \beta$ by specifying $m, n$, $p$ to see how our parametrization of HP can reproduces some particular models. 
Table-2

\begin{tabular}{|c|l|l|l|l|}
\hline Models & \multicolumn{1}{|c|}{$\begin{array}{c}\text { Specific Values of } \\
m, n, p\end{array}$} & \multicolumn{1}{c|}{$\begin{array}{c}\text { HP } \\
H(t)\end{array}$} & \multicolumn{1}{c|}{$\begin{array}{c}\text { SF } \\
a(t)\end{array}$} & \multicolumn{1}{c|}{$q(t)$} \\
\hline I & $m=0, p=0, \forall n$ & $\beta$ & $C e^{\beta t}$ & -1 \\
\hline II & $m=-1, p=0, \forall n$ & $\frac{\beta}{t}$ & $C t^{\beta}$ & $-1+\frac{1}{\beta}$ \\
\hline III & $m=0, p=1, n=1$ & $\frac{\beta}{t+\alpha}$ & $C(t+\alpha)^{\beta}$ & $-1+\frac{1}{\beta}$ \\
\hline IV & $m=1, p=0, \forall n$ & $\beta t$ & $C e^{\beta \frac{t^{2}}{2}}$ & $-1-\frac{1}{\beta} \frac{1}{t^{2}}$ \\
\hline V & $m=0, p=1, n=2$ & $\frac{\beta}{t^{2}+\alpha}$ & $C e^{\frac{\beta}{\sqrt{\alpha}} \tan ^{-1} \frac{t}{\sqrt{\alpha}}}$ & $-1+\frac{2}{\beta} t$ \\
\hline VI & $m=0, p=\frac{1}{2}, n=1$ & $\frac{\beta}{\sqrt{t+\alpha}}$ & $C e^{2 \beta \sqrt{t+\alpha}}$ & $-1+\frac{1}{2 \beta} \frac{1}{\sqrt{t+\alpha}}$ \\
\hline VII & $m=0, p=\frac{1}{2}, n=2$ & $\frac{\beta}{\sqrt{t^{2}+\alpha}}$ & $C\left(t+\sqrt{t^{2}+\alpha}\right)^{\beta}$ & $-1+\frac{1}{\beta} \frac{t}{\sqrt{t^{2}+\alpha}}$ \\
\hline VIII & $m=1, p=1, n=1$ & $\frac{\beta t}{t+\alpha}$ & $C e^{\beta t}(t+\alpha)^{-\alpha \beta}$ & $-1-\frac{\alpha}{\beta} \frac{1}{t^{2}}$ \\
\hline IX & $m=1, p=1, n=2$ & $\frac{\beta t}{t^{2}+\alpha}$ & $C\left(t^{2}+\alpha\right)^{\frac{\beta}{2}}$ & $-1+\frac{1}{\beta}-\frac{\alpha}{\beta} \frac{1}{t^{2}}$ \\
\hline $\mathrm{X}$ & $m=1, p=\frac{1}{2}, n=2$ & $\frac{\beta t}{\sqrt{t^{2}+\alpha}}$ & $C e^{\beta \sqrt{t^{2}+\alpha}}$ & $-1-\frac{\alpha}{\beta} \frac{1}{t^{2} \sqrt{t^{2}+\alpha}}$ \\
\hline $\mathrm{XI}$ & $m=-1, p=1, n=1$ & $\frac{\beta}{t(t+\alpha)}$ & $C\left(\frac{t}{t+\alpha}\right)^{\frac{\beta}{\alpha}}$ & $-1+\frac{\alpha}{\beta}+\frac{2}{\beta} t$ \\
\hline $\mathrm{XII}$ & $m=-1, p=1, n=2$ & $\frac{\beta}{t\left(t^{2}+\alpha\right)}$ & $C\left(\frac{t^{2}}{t^{2}+\alpha}\right)^{\frac{\beta}{2 \alpha}}$ & $-1+\frac{\alpha}{\beta}+\frac{3}{\beta} t^{2}$ \\
\hline
\end{tabular}

In table-2, we see $\Lambda C D M$ model (model-I, where $\beta$ plays the role of $\Lambda$ ), power law cosmology (PLC) [71] (model-II), Berman's model of constant deceleration parameter (BM) 72] (model-III with $\frac{1}{\beta}=m$ ), Abdel Rahman's model (AR) (model-IX) [73] (with $\beta=1$ ) and its generalized model [74] are obtained here. Model-XI imitate the linearly varying deceleration parameter model (LVDPt) of Akarsu [75] (where $\frac{2}{\beta}=-k$ and $\frac{\alpha}{\beta}=m$ ). Thus, we can see all these models come under our scheme of parametrization of HP for some specific choice of model parameters. We note that, many solutions obtained here are non-singular bouncing solutions, where the bounce occur at some finite value of the scale factor $a(t)$.

For $\alpha=0$, the form of $H(t)$ becomes $H(t)=\beta t^{\gamma},(\gamma=(m-n p)$ is a new constant) giving the same result as $p=0$ (Models-I,II,IV). The case for negative $\alpha$ is that for which our main ansatz (8) will take the form $H(t)=\frac{\beta t^{m}}{\left(t^{n}-\alpha\right)^{p}}, \alpha>0$. In this case the behavior of scale factor will differ greatly in some models and so as dynamics e.g. with this form of HP, all the models $(p \neq 0)$ have collapsing nature at $t=\alpha^{\frac{1}{n}}$. If we take $H(t)=\frac{\beta t^{m}}{\left(\alpha-t^{n}\right)^{p}}, \alpha>t$ then this form of HP will lead to models with future singularity at $t=\alpha^{\frac{1}{n}}$. One can study these future singularities for different models so obtained. Modifying the form of HP to $H(t)=\frac{\beta t^{m}+\eta}{\left(\alpha-t^{n}\right)^{p}}, \alpha>t$ and $\eta$ is another parameter then for $p=0$, one can obtain the hybrid scale factor cosmology [76], [77] and for $p=-1$ one can obtain some results discussed by Nojiri and Odintsov [60]. We should mention here that different evolution of the scale factor with a variable cosmological term $\Lambda$ is studied extensively by Overduin and Cooperstock in [78]. Similarly, our parametrization of $H(t)$ also gives rise to different evolutions of scale factor that is being studied in this work.

We shall make note that, $\alpha \& \beta$ are two model parameters and the dynamics of obtained models (in table-2) or the behavior of cosmological parameters $a(t), H(t), q(t), \rho(t), w(t)$ all heavily depend on these two. In the next section we shall discuss the behavior of different cosmological parameters in view of the positive value of the model parameters $\alpha \& \beta$ and 
discuss their analytical bounds. A lot of studies have been done on model-I $(\Lambda \mathrm{CDM})$, model-II (PLC) and model-III (BM). So, we do not dwell in these known models and try to explore the other models only i.e. models-IV-XII.

\section{Dynamics of models}

The expressions for the scale factor, Hubble parameter and deceleration parameter for model-IV-XII are given in table-2. For the positive values of $\alpha, \beta$, their behavior near the singularities (at $t=0$ and $t \rightarrow \infty$ ) are obtained as

Table-3

\begin{tabular}{|l|l|l|l|l|l|l|l|l|l|l|}
\hline $\mathrm{CP} \downarrow$ & models & $\mathrm{IV}$ & $\mathrm{V}$ & $\mathrm{VI}$ & $\mathrm{VII}$ & $\mathrm{VIII}$ & $\mathrm{IX}$ & $\mathrm{X}$ & $\mathrm{XI}$ & $\mathrm{XII}$ \\
\hline \multirow{3}{*}{$a(t)$} & $t=0$ & $C$ & $C$ & $C e^{2 \beta \sqrt{\alpha}}$ & $C \alpha^{\beta / 2}$ & $C \alpha^{-\alpha \beta}$ & $C \alpha^{\beta / 2}$ & $C e^{\beta \sqrt{\alpha}}$ & 0 & 0 \\
\cline { 2 - 11 } & $t \rightarrow \infty$ & $\infty$ & $C e^{\frac{\pi \beta}{2 \sqrt{\alpha}}}$ & $\infty$ & $\infty$ & $\infty$ & $\infty$ & $\infty$ & $C$ & $C$ \\
\hline \multirow{2}{*}{$H(t)$} & $t=0$ & 0 & $\frac{\beta}{\alpha}$ & $\frac{\beta}{\sqrt{\alpha}}$ & $\frac{\beta}{\sqrt{\alpha}}$ & 0 & 0 & 0 & $\infty$ & $\infty$ \\
\cline { 2 - 11 } & $t \rightarrow \infty$ & $\infty$ & 0 & 0 & 0 & $\beta$ & 0 & $\beta$ & 0 & 0 \\
\hline \multirow{2}{*}{$q(t)$} & $t=0$ & $-\infty$ & -1 & $-1+\frac{1}{2 \beta \sqrt{\alpha}}$ & -1 & $-\infty$ & $-\infty$ & $-\infty$ & $-1+\frac{\alpha}{\beta}$ & $-1+\frac{\alpha}{\beta}$ \\
\cline { 2 - 10 } & $t \rightarrow \infty$ & -1 & $+\infty$ & -1 & $-1+\frac{1}{\beta}$ & -1 & $-1+\frac{1}{\beta}$ & -1 & $+\infty$ & $+\infty$ \\
\hline
\end{tabular}

From the above table we can see that the models-IV-X are free from initial singularity and starts with a finite initial radius while models-XI,XII have big bang origin. As $t \rightarrow \infty$ models-IV,VI,VII,VIII,IX,X diverge while the scale factor takes finite values in models-V,XI,XII. Models-IV,VI,VIII,X collapse in near future showing these models have finite time singularity but the singularity can be delayed by larger (smaller in case of model-IV) values $\beta$. Similarly, looking at the values of HP and DP near singularities, we can conclude that in case of modelsIV,VIII,IX,X, the Universe starts with zero velocity and infinite acceleration. In case of ModelV,VI,VII the Universe starts with finite velocity and finite acceleration while in models-XI,XII the Universe starts with infinite velocity and finite acceleration. The rate of initial velocities and initial accelerations for these models depend upon the choice of model parameters $\alpha \& \beta$. We can observe that in the models-V,XI,XII, the Universe ceases as $t \rightarrow \infty$ where the velocity becomes zero and DP becomes $+\infty$ (no acceleration).

As the observations reveal that the total energy budget of the Universe is dominated by DE $(\sim 70 \%)$ and accelerates the expansion of the Universe while non-relativistic baryonic and cold dark matter dominated the total energy budget at earlier times, causing the deceleration. The cosmological deceleration-acceleration transition occurred at some time where $q=0$ (or $\ddot{a}=0$ ). So, at present theorists take interest in modelling the Universe with phase transition from early deceleration to present acceleration. These kinds of models are considered as viable models as there is an obvious provision for the structure formation in the Universe during the decelerated phase and also they can explain the result of observation of Type Ia supernovae at present. On the other hand, before the discovery of late time acceleration, theorists were taking interest in modelling the Universe with early inflation and late time deceleration of the Universe as early inflation is necessary to explain the origin of the large scale structure of the cosmos. So, the 
deceleration-acceleration phase transition is important in current picture while the accelerationdeceleration phase transition is important in the very early Universe. In conclusion, we can say a model which has initial acceleration, middle deceleration and late-time acceleration scenario can be treated as a better model that can explain all the phenomena explained by observations.

Out of the twelve models listed in table-2, the DP comes out to be constant in models-I,II,III where as the DP is time-dependent in models-IV-XII. For $\alpha, \beta>0$, models-IV,VIII,X exhibit eternal acceleration and models-V,VII,IX,XI,XII show transition from initial acceleration to deceleration; or may accelerate for ever for certain choice of $\alpha$ and $\beta$. Only model-VI shows a phase transition from deceleration to acceleration. The various cases in view of phase transition are analyzed in the following table.

Table- 4

\begin{tabular}{|c|l|c|l|}
\hline Models & Transition type & Phase transition time $t_{t r}$ & constrain on $\alpha, \beta$ \\
\hline IV & Ever accelerating & - & $\alpha, \beta>0$ \\
\hline V & Acceleration $\rightarrow$ Deceleration & $\frac{\beta}{2}$ & $\alpha, \beta>0$ \\
\hline VI & Deceleration $\rightarrow$ Acceleration & $\frac{1}{4 \beta^{2}}-\alpha$ & $\alpha, \beta>0, \beta \sqrt{\alpha}<\frac{1}{2}$ \\
& Ever accelerating & - & $\alpha, \beta>0, \beta \sqrt{\alpha}>\frac{1}{2}$ \\
\hline VII & Acceleration $\rightarrow$ Deceleration & $\frac{\sqrt{\alpha} \beta}{\sqrt{1-\beta^{2}}}$ & $\alpha, \beta>0, \beta \sqrt{1+\alpha}>1$ \\
& Ever accelerating & - & $\alpha, \beta>0, \beta \sqrt{1+\alpha}<1$ \\
\hline VIII & Ever accelerating & - & $\alpha, \beta>0$ \\
\hline IX & Acceleration $\rightarrow$ Deceleration & $\sqrt{\frac{\alpha}{1-\beta}}$ & $\alpha, \beta>0, \beta+\alpha>1$ \\
& Ever accelerating & - & $\alpha, \beta>0, \beta+\alpha<1$ \\
\hline $\mathrm{X}$ & Ever accelerating & $-\frac{\beta-\alpha}{2}$ & $\alpha, \beta>0, \beta>\alpha$ \\
\hline $\mathrm{XI}$ & Acceleration $\rightarrow$ Deceleration & - & $\alpha, \beta>0, \beta<\alpha$ \\
\hline $\mathrm{XII}$ & Ever Decelerating & $\sqrt{\frac{\beta-\alpha}{3}}$ & $\alpha, \beta>0, \beta>\alpha$ \\
& Acceleration $\rightarrow$ Deceleration & - & $\alpha, \beta<0, \beta<\alpha$ \\
\hline
\end{tabular}

Observations suggest the present value of DP is somewhere in the neighborhood of -0.55 . So, to have a better understanding of evolutions of DP over time for all the models-IV-XII, we plot them (see figure-1) by choosing the values of $\alpha$ and $\beta$ appropriately such that the present value of DP $q_{0}$ will be in the neighborhood -0.55 or with a very small positive value of $q_{0}$ (for decelerating models). It may be noted that the observations favour accelerating models but the decelerating models are also in agreement with these observations [79]. The decelerating models also show nice fit to some data even with zero cosmological constant if one considers the extinction of light by the metallic dust ejected from the supernovae explosions [79]. With some independent analysis, we have chosen the values of $\alpha$ and $\beta$ in model-VI such that $q_{0} \approx-0.55$. This gives the phase transition time from deceleration to acceleration is around $t_{t r} \approx 3$. The time evolution of $q(t)$ for models-IV-XII are 

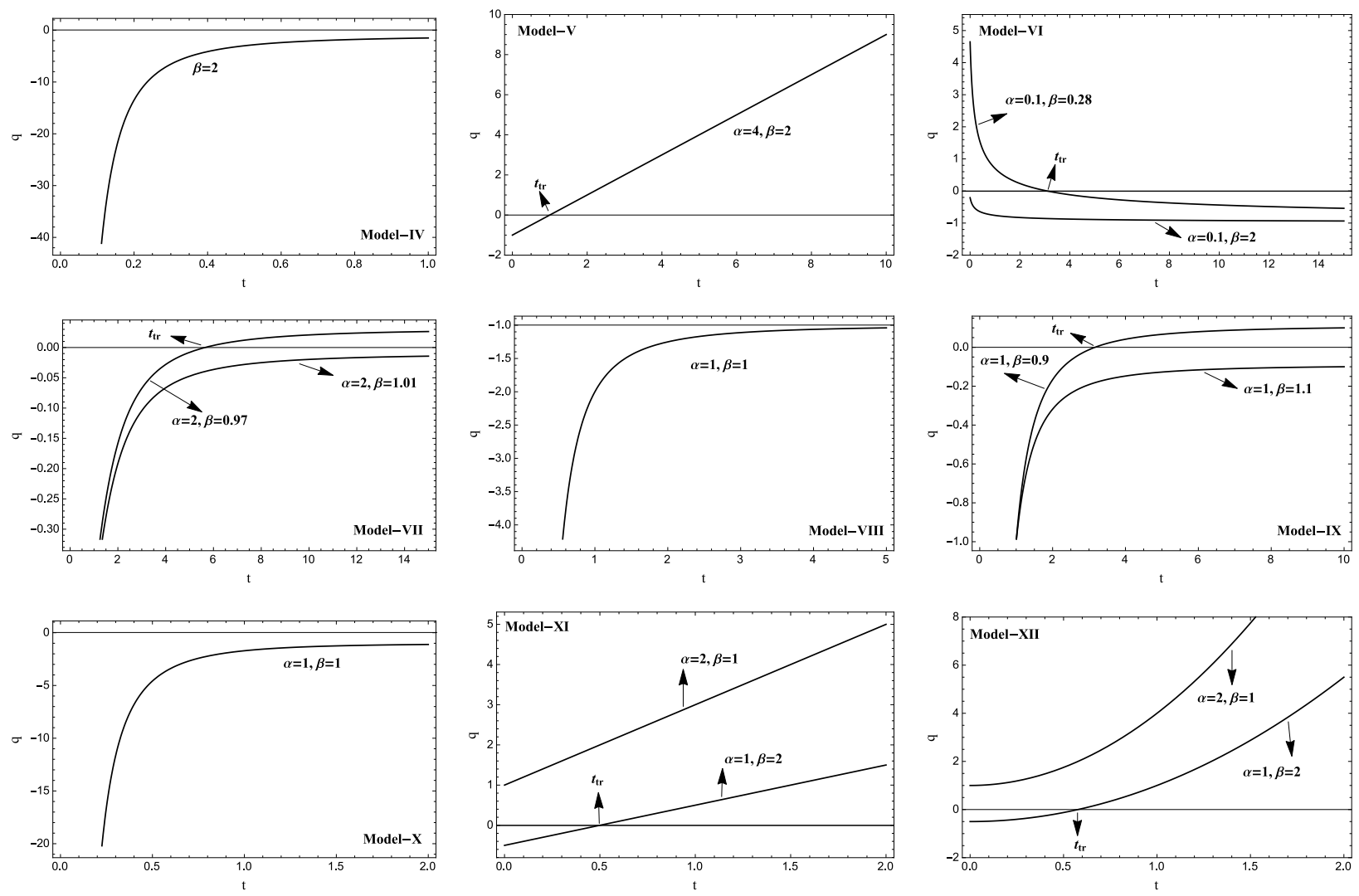

Figure 1: Plots for the Deceleration parameter for models-IV-XII. We can see model-IV (with $\beta=2$ ), model-VIII (with $\alpha=1, \beta=1$ ), model-X (with $\alpha=1, \beta=1$ ) show eternal acceleration while model-V (with $\alpha=4, \beta=2$ ), model-VII (with $\alpha=2, \beta=0.97$ ), model-IX (with $\alpha=$ $1, \beta=0.9$ ), model-XI (with $\alpha=1, \beta=2$ ), model-XII (with $\alpha=1, \beta=2$ ) show transition from acceleration to deceleration. Only model-VI (with $\alpha=0.1, \beta=0.28$ ) shows transition from deceleration to acceleration. We can also see that the model-VI (with $\alpha=0.1, \beta=2$ ), model-VII (with $\alpha=2, \beta=1.01$ ), model-IX (with $\alpha=1, \beta=1.1$ ) show eternal acceleration and model-XI (with $\alpha=2, \beta=1$ ), model-XII (with $\alpha=2, \beta=1$ ) show eternal deceleration as discussed in table- 4 . For the plots we have chosen the units suitably e.g. In case of model-IV, the time axis is scaled such that 0.1 time unit $=1$ billion years while in model- $\mathrm{V}$, the time axis is scaled such that 1 time unit $=1$ billion years and so on. 
The other cosmological parameters $\rho(t), \rho_{\text {eff }}(t), w_{\text {eff }}(t)$ for models-IV-XII are obtained as

\section{Model-IV}

$\rho(t)=\left[D C^{-3(1+w)}\right] e^{-3(1+w) \beta \frac{t^{2}}{2}}$

$\rho_{e f f}(t)=3 M_{P l}^{2}\left[\beta^{2} t^{2}+\frac{k}{C^{2}} e^{-\beta t^{2}}\right]$

$w_{e f f}(t)=\frac{1}{3} \frac{\left(-3-\frac{2}{\beta} \frac{1}{t^{2}}\right) \beta^{2} t^{2}-\frac{k}{C^{2}} e^{-\beta t^{2}}}{\left[\beta^{2} t^{2}+\frac{k}{C^{2}} e^{-\beta t^{2}}\right]}$

\section{Model-VI}

$\rho(t)=\left[D C^{-3(1+w)}\right] e^{-6(1+w) \beta \sqrt{t+\alpha}}$

$\rho_{\text {eff }}(t)=3 M_{P l}^{2}\left[\frac{\beta^{2}}{t+\alpha}+\frac{k}{C^{2}} e^{-4 \beta \sqrt{t+\alpha}}\right]$

$w_{e f f}(t)=\frac{1}{3} \frac{\left(-3+\frac{1}{\beta} \frac{1}{\sqrt{t+\alpha}}\right) \frac{\beta^{2}}{t+\alpha}-\frac{k}{C^{2}} e^{-4 \beta \sqrt{t+\alpha}}}{\frac{\beta^{2}}{t+\alpha}+\frac{k}{C^{2}} e^{-4 \beta \sqrt{t+\alpha}}}$

\section{Model-VIII}

$\rho(t)=\left[D C^{-3(1+w)}\right] e^{-3(1+w) \beta t}(t+\alpha)^{3(1+w) \alpha \beta}$

$\rho_{e f f}(t)=3 M_{P l}^{2}\left[\frac{\beta^{2} t^{2}}{(t+\alpha)^{2}}+\frac{k}{C^{2} e^{2 \beta t}(t+\alpha)^{-2 \alpha \beta}}\right]$

$w_{e f f}(t)=\frac{1}{3} \frac{\left(-3-\frac{2 \alpha}{\beta} \frac{1}{t^{2}}\right) \frac{\beta^{2} t^{2}}{(t+\alpha)^{2}}-\frac{k}{C^{2} e^{2 \beta t}(t+\alpha)^{-2 \alpha \beta}}}{\frac{\beta^{2} t^{2}}{(t+\alpha)^{2}}+\frac{k}{C^{2} e^{2 \beta t}(t+\alpha)^{-2 \alpha \beta}}}$

\section{Model-X}

$\rho(t)=\left[D C^{-3(1+w)}\right] e^{-3(1+w) \beta \sqrt{t^{2}+\alpha}}$

$\rho_{e f f}(t)=3 M_{P l}^{2}\left[\frac{\beta^{2} t^{2}}{\left(t^{2}+\alpha\right)}+\frac{k}{C^{2} e^{2 \beta \sqrt{t^{2}+\alpha}}}\right]$

$w_{e f f}(t)=\frac{1}{3} \frac{\left(-3-\frac{2 \alpha}{\beta} \frac{1}{t^{2} \sqrt{t^{2}+\alpha}}\right) \frac{\beta^{2} t^{2}}{t^{2}+\alpha}-\frac{k}{C^{2} e^{2 \beta \sqrt{t^{2}+\alpha}}}}{\frac{\beta^{2} t^{2}}{t^{2}+\alpha}+\frac{k}{C^{2} e^{2 \beta} \sqrt{t^{2}+\alpha}}}$

\section{Model-XII}

$$
\begin{aligned}
& \rho(t)=\left[D C^{-3(1+w)}\right]\left(\frac{t^{2}}{t^{2}+\alpha}\right)^{-3(1+w) \frac{\beta}{2 \alpha}} \\
& \rho_{e f f}(t)=3 M_{P l}^{2}\left[\frac{\beta^{2}}{t^{2}\left(t^{2}+\alpha\right)^{2}}+\frac{k}{C^{2}\left(\frac{t^{2}}{t^{2}+\alpha}\right)^{\frac{\beta}{\alpha}}}\right] \\
& w_{e f f}(t)=\frac{1}{3} \frac{\left(-3+\frac{2 \alpha}{\beta}+\frac{6}{\beta} t^{2}\right) \frac{\left.\beta^{2}\right)}{t^{2}\left(t^{2}+\alpha\right)^{2}}-\frac{k}{C^{2}\left(\frac{t^{2}}{t^{2}+\alpha}\right)^{\frac{\beta}{\alpha}}}}{\frac{\beta^{2}}{t^{2}\left(t^{2}+\alpha\right)^{2}}+\frac{k}{C^{2}\left(\frac{t^{2}}{t^{2}+\alpha}\right)^{\frac{\beta}{\alpha}}}}
\end{aligned}
$$

\section{Model-VII}

$\rho(t)=\left[D C^{-3(1+w)}\right]\left(t+\sqrt{t^{2}+\alpha}\right)^{-3(1+w) \beta}$

$\rho_{e f f}(t)=3 M_{P l}^{2}\left[\frac{\beta^{2}}{t^{2}+\alpha}+\frac{k}{C^{2}\left(t+\sqrt{t^{2}+\alpha}\right)^{2 \beta}}\right]$

$w_{e f f}(t)=\frac{1}{3} \frac{\left(-3+\frac{2}{\beta} \frac{t}{\sqrt{t^{2}+\alpha}}\right) \frac{\beta^{2}}{t^{2}+\alpha}-\frac{k}{C^{2}\left(t+\sqrt{t^{2}+\alpha}\right)^{2 \beta}}}{\frac{\beta^{2}}{t^{2}+\alpha}+\frac{k}{C^{2}\left(t+\sqrt{t^{2}+\alpha}\right)^{2 \beta}}}$

\section{Model-IX}

$\rho(t)=\left[D C^{-3(1+w)}\right]\left(t^{2}+\alpha\right)^{-3(1+w) \frac{\beta}{2}}$

$\rho_{e f f}(t)=3 M_{P l}^{2}\left[\frac{\beta^{2} t^{2}}{\left(t^{2}+\alpha\right)^{2}}+\frac{k}{C^{2}\left(t^{2}+\alpha\right)^{\beta}}\right]$

$w_{e f f}(t)=\frac{1}{3} \frac{\left(-3+\frac{2}{\beta}-\frac{2 \alpha}{\beta} \frac{1}{t^{2}}\right) \frac{\beta^{2} t^{2}}{\left(t^{2}+\alpha\right)^{2}}-\frac{k}{C^{2}\left(t^{2}+\alpha\right)^{\beta}}}{\frac{\beta^{2} t^{2}}{\left(t^{2}+\alpha\right)^{2}}+\frac{k}{C^{2}\left(t^{2}+\alpha\right)^{\beta}}}$

\section{Model-XI}

$\rho(t)=\left[D C^{-3(1+w)}\right]\left(\frac{t}{t+\alpha}\right)^{-3(1+w) \frac{\beta}{\alpha}}$

$\rho_{e f f}(t)=3 M_{P l}^{2}\left[\frac{\beta^{2}}{t^{2}(t+\alpha)^{2}}+\frac{k}{C^{2}\left(\frac{t}{t+\alpha}\right)^{2 \frac{\beta}{\alpha}}}\right]$

$w_{e f f}(t)=\frac{1}{3} \frac{\left(-3+\frac{2 \alpha}{\beta}+\frac{2}{\beta} t\right) \frac{\beta^{2}}{t^{2}(t+\alpha)^{2}}-\frac{k}{C^{2}\left(\frac{t}{t+\alpha}\right)^{2 \frac{\beta}{\alpha}}}}{\frac{\beta^{2}}{t^{2}(t+\alpha)^{2}}+\frac{k}{C^{2}\left(\frac{t}{t+\alpha}\right)^{2 \frac{\beta}{\alpha}}}}$ 


\subsection{Negative $\beta$ consideration}

We discuss the possibility of taking negative value of $\beta$ together with negative $\alpha$ in certain models giving rise to some new cosmologies. In this work, we consider negative $\beta$ together with negative $\alpha$ in models-XI,XII only. In other models one can work out for negative $\alpha, \beta$ in models-III,V,VIII,IX where $\alpha>t$. This kind of analysis have been done by Nojiri and Odintsov [69], [70], 60] to study the future finite time singularity where they have taken $\alpha=t_{s} \rightarrow$ future singularity time.

So, for negative $\alpha, \beta$ we obtain the cosmological parameters for models-XI,XII as

\section{Model-XI*}

$$
\begin{aligned}
& H(t)=\frac{\beta}{t(\alpha-t)} \\
& a(t)=C\left(\frac{t}{\alpha-t}\right)^{\frac{\beta}{\alpha}} \\
& q(t)=-1+\frac{\alpha}{\beta}-\frac{2}{\beta} t \\
& \rho(t)=\left[D C^{-3(1+w)}\right]\left(\frac{t}{\alpha-t}\right)^{-3(1+w) \frac{\beta}{\alpha}} \\
& \rho_{e f f}(t)=3 M_{P l}^{2}\left[\frac{\beta^{2}}{t^{2}(\alpha-t)^{2}}+\frac{k}{C^{2}\left(\frac{t}{\alpha-t}\right)^{2 \frac{\beta}{\alpha}}}\right] \\
& \frac{1}{3} \frac{\left(-3+\frac{2 \alpha}{\beta}-\frac{4}{\beta} t\right) \frac{\beta^{2}}{t^{2}(\alpha-t)^{2}}-\frac{k}{C^{2}\left(\frac{t}{\alpha-t}\right)^{2 \frac{\beta}{\alpha}}}}{\frac{\beta^{2}}{t^{2}(\alpha-t)^{2}}+\frac{k}{C^{2}\left(\frac{t}{\alpha-t}\right)^{2 \frac{\beta}{\alpha}}}}
\end{aligned}
$$

Model-XII*

$$
\begin{aligned}
& H(t)=\frac{\beta}{t\left(\alpha-t^{2}\right)} \\
& a(t)=C\left(\frac{t^{2}}{\alpha-t^{2}}\right)^{\frac{\beta}{2 \alpha}} \\
& q(t)=-1+\frac{\alpha}{\beta}-\frac{3}{\beta} t^{2} \\
& \rho(t)=\left[D C^{-3(1+w)}\right]\left(\frac{t^{2}}{\alpha-t^{2}}\right)^{-3(1+w) \frac{\beta}{2 \alpha}} \\
& \rho_{\text {eff }}(t)=3 M_{P l}^{2}\left[\frac{\beta^{2}}{t^{2}\left(\alpha-t^{2}\right)^{2}}+\frac{k}{C^{2}\left(\frac{t^{2}}{\alpha-t^{2}}\right)^{\frac{\beta}{\alpha}}}\right] \\
& \frac{\left(-3+\frac{2 \alpha}{\beta}-\frac{6}{\beta} t^{2}\right) \frac{\beta^{2}}{t^{2}\left(\alpha-t^{2}\right)^{2}}-\frac{k}{C^{2}\left(\frac{t^{2}}{\alpha-t^{2}}\right)^{\frac{\beta}{\alpha}}}}{\frac{\beta^{2}}{t^{2}\left(\alpha-t^{2}\right)^{2}}+\frac{k}{C^{2}\left(\frac{t^{2}}{\alpha-t^{2}}\right)^{\frac{\beta}{\alpha}}}}
\end{aligned}
$$

For both these models, Hubble parameter and scale factor both diverge in finite time and show big rip singularity in near future.

\subsection{Observational constrain for models showing $\mathrm{DEC} \rightarrow \mathrm{ACC}$ transi- tion}

To constrain the model parameters $\alpha$ and $\beta$ and to compare our results with observation, we also re-write the DP and HP that are given as functions of cosmic time $t$, in terms of redshift $z\left(=\frac{a_{0}}{a}-1\right.$, where $a_{0}$ is the value of scale factor at present time $\left.t=t_{0}\right)$ using the relation between $t$ and $z$ for the models with deceleration $\rightarrow$ acceleration transition i.e. for models-VI,XI*,XII*

\section{Model-VI}

$$
\begin{aligned}
& t(z)=-\alpha+\left[\sqrt{t_{0}+\alpha}-\frac{1}{2 \beta} \ln (1+z)\right]^{2} \\
& q(z)=-1+\frac{1}{\beta}\left[\sqrt{t_{0}+\alpha}-\frac{1}{2 \beta} \ln (1+z)\right]^{-1} \\
& H(z)=H_{0}\left[1-\frac{\ln (1+z)}{2 \beta \sqrt{t_{0}+\alpha}}\right]^{-1} \\
& \text { Model-XI* } \\
& t(z)=\alpha\left[1+\left(\frac{\alpha}{t_{0}}-1\right)(1+z)^{\frac{\alpha}{\beta}}\right]^{-1} \\
& q(z)=-1+\frac{\alpha}{\beta}-\frac{2 \alpha}{\beta}\left[1+\left(\frac{\alpha}{t_{0}}-1\right)(1+z)^{\frac{\alpha}{\beta}}\right]^{-1} \\
& H(z)=\frac{H_{0} t_{0}^{2}}{\alpha^{2}}\left\{(1+z)^{-\frac{\alpha}{2 \beta}}+\left(\frac{\alpha}{t_{0}}-1\right)(1+z)^{\frac{\alpha}{2 \beta}}\right\}^{2}
\end{aligned}
$$




$$
\begin{aligned}
& t(z)=\sqrt{\alpha}\left[1+\left(\frac{\alpha}{t_{0}^{2}}-1\right)(1+z)^{\frac{2 \alpha}{\beta}}\right]^{-\frac{1}{2}} \\
& q(z)=-1+\frac{\alpha}{\beta}-\frac{3 \alpha}{\beta}\left[1+\left(\frac{\alpha}{t_{0}^{2}}-1\right)(1+z)^{\frac{2 \alpha}{\beta}}\right]^{-1} \\
& H(z)=\frac{H_{0} t_{0}^{3}}{\alpha^{\frac{3}{2}}}\left\{(1+z)^{-\frac{4 \alpha}{3 \beta}}+\left(\frac{\alpha}{t_{0}^{2}}-1\right)(1+z)^{\frac{2 \alpha}{3 \beta}}\right\}^{\frac{3}{2}}
\end{aligned}
$$

We find the observational constraints on both of the model parameters $\alpha$ and $\beta$ to the latest 28 data points of $H(z)$ in the redshift range $0.100 \leqslant z \leqslant 2.3$ (see table- 5 ). The observational data consist of measurements of the Hubble parameter at particular redshifts with the corresponding standard deviations $\left(\sigma_{H}\right)$ given by

Table-5 $\quad$ Hubble parameter vs redshift data

\begin{tabular}{|c|c|c|c|c|c|c|c|}
\hline$z$ & $H(z)\left(\frac{\mathrm{kms}^{-1}}{M p c}\right)$ & $\sigma_{H}\left(\frac{\mathrm{kms}^{-1}}{M p c}\right)$ & $R e f$. & $z$ & $H(z)\left(\frac{k m \mathrm{~s}^{-1}}{M p c}\right)$ & $\sigma_{H}\left(\frac{\mathrm{kms}^{-1}}{M p c}\right)$ & $R e f$. \\
\hline 0.100 & 69 & 12 & {$[80]$} & 0.730 & 97.3 & 7 & {$[83]$} \\
\hline 0.170 & 83 & 8 & {$[80]$} & 0.781 & 105 & 12 & {$[81]$} \\
\hline 0.179 & 75 & 4 & {$[81]$} & 0.875 & 125 & 17 & {$[81]$} \\
\hline 0.199 & 75 & 5 & {$[81]$} & 0.880 & 90 & 40 & {$[84]$} \\
\hline 0.270 & 77 & 14 & {$[80]$} & 0.900 & 117 & 23 & {$[80]$} \\
\hline 0.320 & 79.2 & 5.6 & {$[82]$} & 1.037 & 154 & 20 & {$[81]$} \\
\hline 0.352 & 83 & 14 & {$[81]$} & 1.300 & 168 & 17 & {$[80]$} \\
\hline 0.400 & 95 & 17 & {$[80]$} & 1.363 & 160 & 33.6 & {$[85]$} \\
\hline 0.440 & 82.6 & 7.8 & {$[83]$} & 1.430 & 177 & 18 & {$[80]$} \\
\hline 0.480 & 97 & 62 & {$[84]$} & 1.530 & 140 & 14 & {$[80]$} \\
\hline 0.570 & 100.3 & 3.7 & {$[82]$} & 1.750 & 202 & 40 & {$[80]$} \\
\hline 0.593 & 104 & 13 & {$[81]$} & 1.965 & 186.5 & 50.4 & {$[85]$} \\
\hline 0.600 & 87.9 & 6.1 & {$[83$} & 2.340 & 222 & 7 & {$[86$} \\
\hline 0.680 & 92 & 8 & {$[81]$} & 2.360 & 226 & 8 & {$[87]$} \\
\hline
\end{tabular}

To complete the data set, we take $H_{0}=67.8 \mathrm{Km} / \mathrm{s} / \mathrm{Mpc}$. The mean values of model parameters $\alpha$ and $\beta$ are determined by minimizing

$$
\chi_{O H D}^{2}\left(p_{s}\right)=\sum_{i=1}^{28} \frac{\left[H_{t h}\left(p_{s} ; z_{i}\right)-H_{o b s}\left(z_{i}\right)\right]^{2}}{\sigma_{H\left(z_{i}\right)}^{2}}
$$

where $p_{s}$ denotes the parameters of the model, $H_{t h}$ is the theoretical (model based) value for the Hubble parameter, $H_{o b s}$ is the observed one, $\sigma_{H\left(z_{i}\right)}$ is the standard error in the observed value, and the summation runs over 28 observational data points at redshifts $z_{i}$.

From our analysis, the model-VI show a poor fit for higher redshifts (not shown), but models$\mathrm{XI}^{*} \& \mathrm{XII}^{*}$ show nice fit to the Hubble data compared with $\Lambda C D M$ model and are shown in figure-2. The likelihood contours in the $\alpha-\beta$ plane with $1 \sigma$ and $2 \sigma$ error are also obtained for these models and are shown in figure-3. 

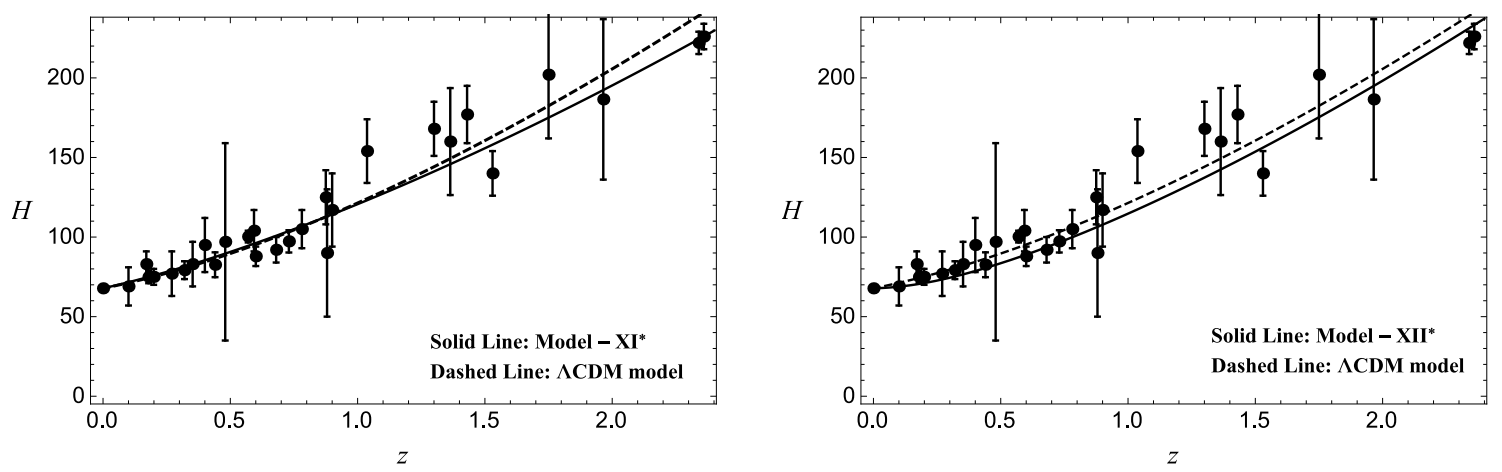

Figure 2: This figure corresponds to the latest $H(z)$ data with error bars. In both the plots, solid lines corresponds to the best fitted behavior for model-XI* \& XII* and dotted lines corresponds to $\Lambda C D M$ model. $H(z)$ is expressed in unit of $\mathrm{Km} / \mathrm{s} / \mathrm{Mpc}$.
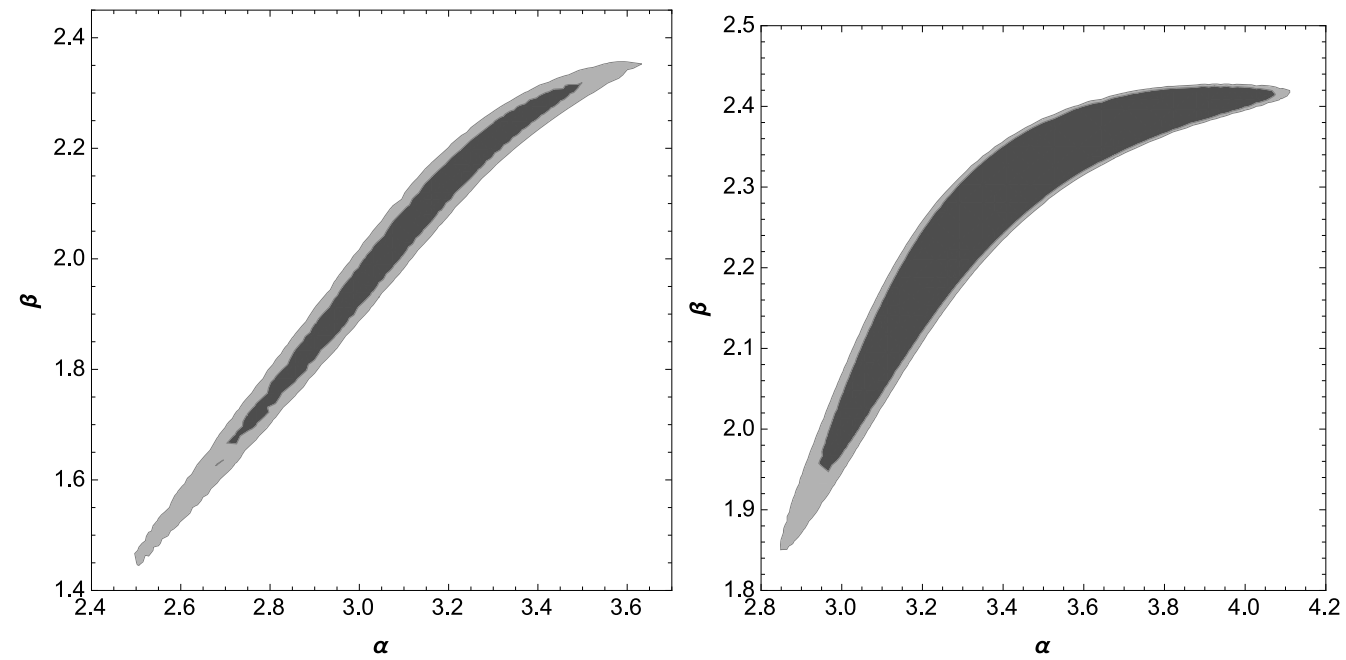

Figure 3: This figure shows the plots for $1 \sigma$ (dark shaded) and $2 \sigma$ (light shaded) likelihood contours in the $\alpha-\beta$ planes, obtained for model-XI* \& XII*. 
The best fit values of $\alpha$ and $\beta$ obtained by minimizing the chi-square $\left(\chi^{2}\right)$ with $1 \sigma$ error are obtained as

$$
\begin{array}{lcc} 
& \alpha & \beta \\
\text { Models-XI* } & 3.051_{-0.34}^{+0.45} & 2.0_{-0.35}^{+0.31} \\
\text { Models-XII* } & 3.006_{-0.075}^{+1.05} & 2.0_{-0.045}^{+0.42}
\end{array} \text {. }
$$

With these values of $\alpha$ and $\beta$, we plot $q(z)$ vs $z$ (see figure-4) and $w(z)$ vs $z$ (see figure$5)$ for models-XI* \& XII* for flat $(k=0)$ case. For both the models EoS parameter $w(z)$ crosses the phantom divide line in near future. The transition redshift $z_{t r}$ from deceleration to acceleration and the redshift $z_{p h}$ at which $w(z)$ crosses the phantom divide line are also shown. From the figures we can find $z_{t r} \approx 0.73$ for model-XI* and $z_{t r} \approx 0.58$ for model-XII*. Similarly, the redshift at which $w(z)$ crosses the phantom divide line is $z_{p h} \approx-0.38$ for model-XI* and $z_{p h} \approx-0.01$ for model-XII*.
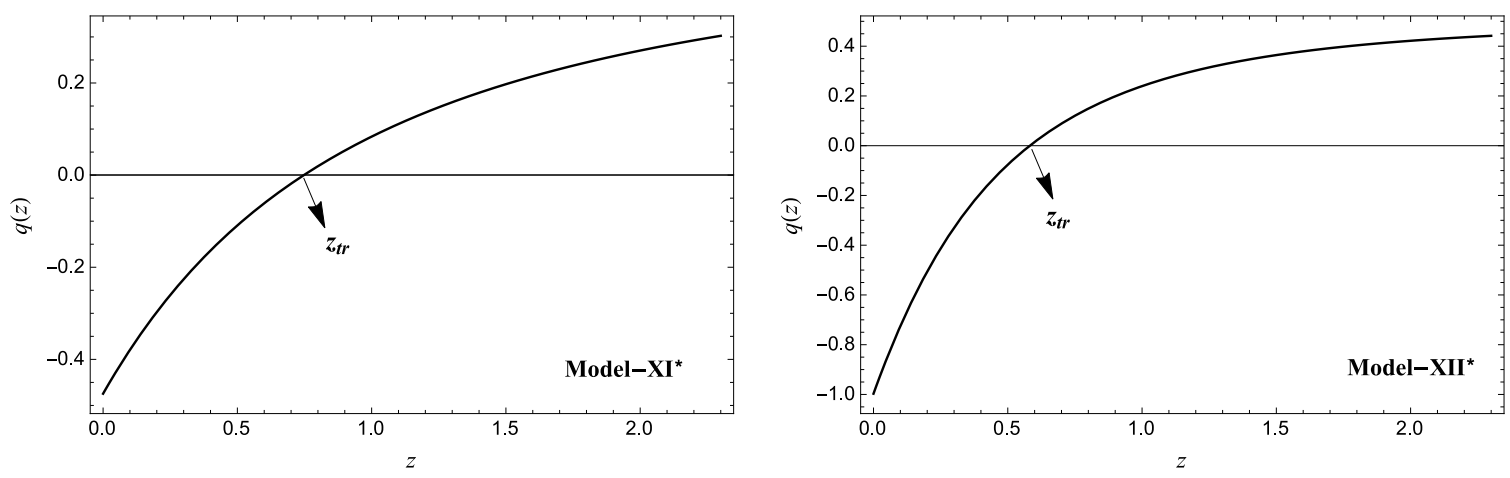

Figure 4: This figure shows plots for the DP $q(z)$ vs redshift $z$ for model-XI* \& XII* showing the transition from deceleration to acceleration.
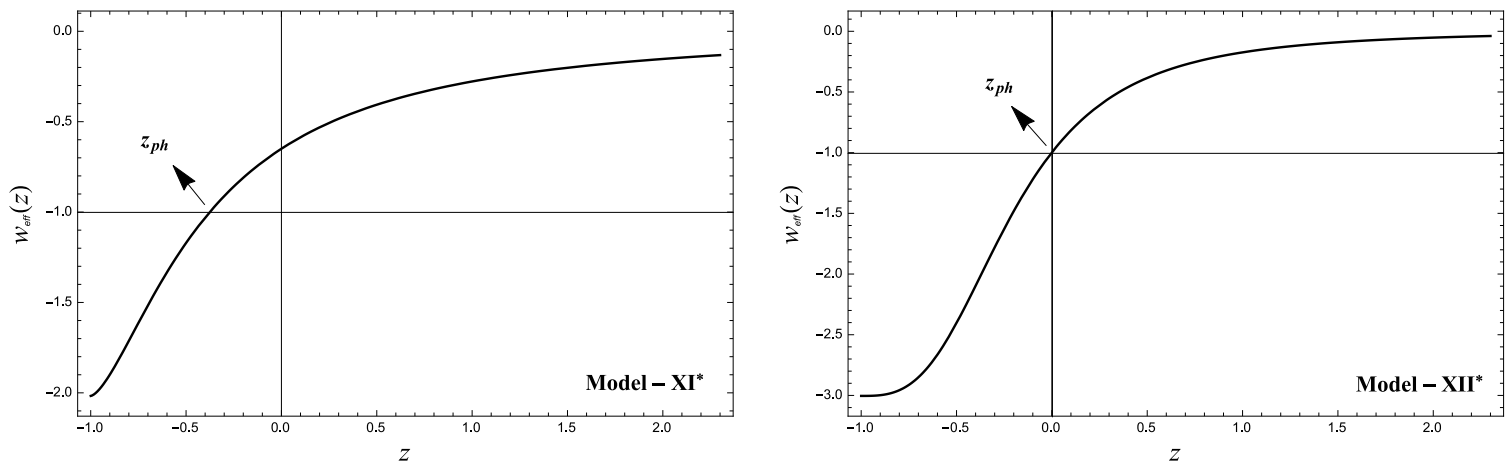

Figure 5: This figure shows plots for the $\operatorname{EoS} w(z)$ vs redshift $z$ for model-XI* \& XII* showing the phantom divide line crossing. 


\section{Conclusion}

In this paper we proposed a convenient and simple parametrization of $H$. For certain choices of model parameters in our scheme, we reproduce several known solutions such as

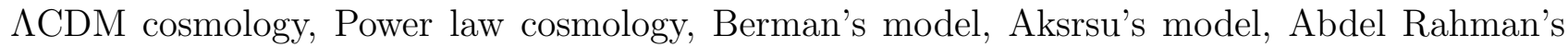
model and others. Thus, our parametrization covers all these models and also produces some new cosmologies. The models under consideration either show transition from deceleration to acceleration or vice versa; in some cases we observed eternal acceleration. The various constrain equations and parametrization related to $a(t), H(t), q(t), \Lambda(t), \rho(t), w(t)$ considered in literature are also summarized in detail. As the present observations agree with deceleration to acceleration transition, we have analyzed three obtained models which exhibit this important feature. Our analysis shows that the model-VI has a poor fit for higher redshifts, but models$\mathrm{XI}^{*} \& \mathrm{XII}^{*}$ show a nice fit with the Hubble data. The likelihood contours in the $\alpha-\beta$ plane obtained for models-XI* \& XII* are shown in figure-3. The best fit values of $\alpha$ and $\beta$ are given by, $\alpha=3.051_{-0.34}^{+0.45} \& \beta=2.0_{-0.35}^{+0.31}$ for models-XI* and $\alpha=3.006_{-0.075}^{+1.05} \& \beta=2.0_{-0.045}^{+0.42}$ for modelsXII*. For these values of $\alpha$ and $\beta$, the evolution of $q(z)$ showing the deceleration to acceleration phase transition and the evolution of $w(z)$ showing the phantom divide line crossing for these models are shown in figure- 4 and figure- 5 respectively.

It is interesting to note that our parametrization for $H$ can give rise to several interesting features and can further be studied in anisotropic Bianchi space-times in the framework of general theory of relativity as well as in modified theories of gravity. In particular it would be interesting to discuss the problem of future singularities in the proposed framework which we deffer to our future investigations.

Acknowledgement The authors wish to thank M. Sami for his useful comments and suggestions throughout the work. Author SKJP wish to thank Department of Atomic Energy (DAE), Government of India for financial support through the post-doctoral fellowship of $N a$ tional Board of Higher Mathematics (NBHM). SKJP is also thankful to J. V. Narlikar, S. Jhingan and R. G. Vishwakarma for discussions on the related theme and thankful to Safia Ahmad and Dharm Veer Singh for their help in some numerical computations.

Appendix-1

Table-6

\begin{tabular}{|c|c|c|}
\hline Variations of Scale factor $(a)$ & Ref. & \multirow{8}{*}{$\begin{array}{l}\text { where } n, \alpha, \beta, \gamma \text { are constants. } \\
t_{0} \text { is the present time. } \\
t_{s} \text { future singularity time. }\end{array}$} \\
\hline$a \sim t^{n}$ & 71, 88 & \\
\hline$a \sim \exp (\beta t)$ & 89 & \\
\hline$a \sim \sinh (\beta t), a \sim \cosh (\beta t)$ & 89 & \\
\hline$a \sim\left(\frac{t}{t_{0}}\right)^{\alpha} e^{\beta\left(\frac{t}{t_{0}}-1\right)}$ & [76] & \\
\hline$a \sim e^{\alpha t} t^{n}$ & 77 & \\
\hline$a(t)=e^{\alpha\left(t-t_{s}\right)^{2(1+\beta)}}$ & 90 & \\
\hline$a(t)=a_{0}\left(\frac{t}{t_{s}-t}\right)^{\gamma}$ & 91 & \\
\hline
\end{tabular}


Table-7

\begin{tabular}{|l|c|}
\hline $\begin{array}{l}\text { Variations of } \\
\text { Energy density }(\rho)\end{array}$ & Ref. \\
\hline$\rho=\rho_{c}$ & {$[92],[93],[94]$} \\
\hline$\rho \sim \theta^{2}$ & {$[95],[96]$} \\
\hline$\rho=\frac{A}{a^{4}} \sqrt{a^{2}+b}$ & {$[97],[98]$} \\
\hline$(\rho+3 p) a^{3}=A$ & {$[99],[100]$} \\
\hline$\rho+p=\rho_{c}$ & {$[101],[102]$} \\
\hline
\end{tabular}

$A, b$ are constants.

Table- 8

\begin{tabular}{|l|c|c|}
\hline Pressure $(p)$ considerations & EoS & Ref. \\
\hline$p=w \rho$ & perfect fluid & \\
\hline$p=w \rho-f(H)$ & viscous fluid & {$[117],[118]$} \\
\hline$p=-\rho-\rho^{\alpha}$ & DE & {$[119]$} \\
\hline$p=w \rho+k \rho^{1+\frac{1}{n}}$ & polytropic & {$[120],[121]$} \\
\hline$p=-(w+1) \frac{\rho^{2}}{\rho_{P}}+w \rho+(w+1) \rho_{\Lambda}$ & quadratic & {$[122]$} \\
\hline$p=-\frac{B}{\rho}$ & Chaplygin Gas & {$[46],[47]$} \\
\hline$p=-\frac{B}{\rho^{\alpha}}$ & generalized CG & {$[123],[124]$} \\
\hline$p=A \rho-\frac{B}{\rho^{\alpha}}$ & modified CG & {$[125,[126]$} \\
\hline$p=A \rho-\frac{B(a)}{\rho^{\alpha}}$ & variable MCG & {$[127]$} \\
\hline$p=A(a) \rho-\frac{B(a)}{\rho^{\alpha}}$ & new variable MCG & {$[128]$} \\
\hline
\end{tabular}

$\rho_{P}$-Plank density $\rho_{\Lambda}$-vacuum density $0 \leqslant w \leqslant 1, k \leqslant 0$, $A>0, B>0$ are constants. $A(a) \& B(a)$ are functions of scale factor.

Note: In literature there exist numerous solutions to Einstein field equations with the ansatz $\sigma^{2} \propto \theta^{2}$, where $\sigma$ is the energy density associated with anisotropy and $\theta$ is the volume expansion scalar in homogeneous anisotropic Bianchi models.

Table-9

\begin{tabular}{|l|c|}
\hline Variations of cosmological constant $(\Lambda)$ & Ref. \\
\hline$\Lambda \sim a^{-n}$ & {$[103,[104],[105]$} \\
\hline$\Lambda \sim H^{n}$ & {$[78,[105,[107]$} \\
\hline$\Lambda \sim \rho$ & {$[105],[106],[108]$} \\
\hline$\Lambda \sim t^{n}$ & {$[78],[105]$} \\
\hline$\Lambda \sim q^{n}$ & {$[78]$} \\
\hline$\Lambda \sim e^{-\beta a}$ & {$[109]$} \\
\hline$\Lambda=\Lambda(T), T$ is Temperature & {$[110]$} \\
\hline$\Lambda \sim C+e^{-\beta t}$ & {$[11]$} \\
\hline$\Lambda=3 \beta H^{2}+\alpha a^{-2}$ & {$[112],[113]$} \\
\hline$\Lambda=\beta \frac{\dot{a}}{a}$ & {$[114,[106]$} \\
\hline$\Lambda=3 \beta H^{2}+\alpha \frac{\ddot{a}}{a}$ & {$[115]$} \\
\hline$\frac{d \Lambda}{d t} \sim \beta \Lambda-\Lambda^{2}$ & {$[116]$} \\
\hline
\end{tabular}

where $n, \alpha, \beta, C$ appearing in the expressions are constants. For a complete set of decay laws of $\Lambda$ one can see [78]. 
Table-10

\begin{tabular}{|l|c|}
\hline Parametrization of DP $(q)$ & Ref. \\
\hline$q=m-1$ & {$[72],[129]$} \\
\hline$q(t)=-\alpha t+m-1$ & {$[75]$} \\
\hline$q(t)=-\frac{\alpha}{t^{2}}+\beta-1$ & {$[130]$} \\
\hline$q(a)=-1-\frac{\alpha a^{\alpha}}{1+a^{\alpha}}$ & {$[131],[132],[133],[134]$} \\
\hline$q(z)=q_{0}+q_{1} z$ & {$[134],[135],[136]$} \\
\hline$q(z)=q_{0}+q_{1} z(1+z)^{-1}$ & {$[137]$} \\
\hline$q(z)=q_{0}+q_{1} z(1+z)\left(1+z^{2}\right)^{-1}$ & {$[134]$} \\
\hline$q(z)=\frac{1}{2}+q_{1}(1+z)^{-2}$ & {$[136]$} \\
\hline$q(z)=q_{0}+q_{1}[1+\ln (1+z)]^{-1}$ & {$[138],[140],[139]$} \\
\hline$q(z)=\frac{1}{2}+\left(q_{1} z+q_{2}\right)(1+z)^{-2}$ & {$[142]$} \\
\hline$q(z)=-1+\frac{3}{2}\left(\frac{(1+z)^{q_{2}}}{q_{1}+(1+z)^{q_{2}}}\right)$ & {$[142]$} \\
\hline$q(z)=-\frac{1}{4}\left[3 q_{1}+1-3\left(q_{1}+1\right)\left(\frac{q_{1} e^{q_{2}(1+z)}-e^{-q_{2}(1+z)}}{q_{1} e^{q_{2}(1+z)}+e^{-q_{2}(1+z)}}\right)\right.$ & {$[143]$} \\
\hline$q(z)=-\frac{1}{4}+\frac{3}{4}\left(\frac{q_{1} e^{q_{2} \frac{z}{\sqrt{1+z}}}-e^{-q_{2} \frac{z}{\sqrt{1+z}}}}{q_{1} e^{\frac{z}{\sqrt{1+z}}}+e^{-q_{2} \frac{z}{\sqrt{1+z}}}}\right)$ & \\
\hline$q(z)=q_{f}+\frac{q_{i}-q_{f}}{1-\frac{q_{i}}{q_{f}}\left(\frac{1+z_{t}}{1+z}\right)^{\frac{1}{\tau}}}$ & {$\left[q_{1}\right.$} \\
\hline
\end{tabular}

$m, \alpha, \beta, q_{0}, q_{1}, q_{2}$ appearing in the above expressions are constants.

Table-11

\begin{tabular}{|l|c|l|}
\hline Parametrization of EoS $(w)$ & Ref. & \\
\hline$w(z)=w_{0}+w_{1} z$ & {$[144,[145]$} & Linear \\
\hline$w(z)=w_{0}+w_{1} \frac{z}{(1+z)^{2}}$ & {$[146]$} & JBP \\
\hline$w(z)=w_{0}+w_{1} \frac{z}{1+z}$ & {$[147],[148]$} & CPL \\
\hline$w(z)=w_{0}+w_{1} \frac{z}{\sqrt{1+z^{2}}}$ & {$[149]$} & sqrt \\
\hline$w(z)=w_{0}+w_{1} \frac{z(1+z)}{1+z^{2}}$ & {$[150]$} & BA \\
\hline$w(z)=w_{0}+w_{1} \frac{z}{1+z^{2}}$ & {$[151]$} & FSLL Model 1 \\
\hline$w(z)=w_{0}+w_{1} \frac{z^{2}}{1+z^{2}}$ & {$[151]$} & FSLL model 2 \\
\hline$w(z)=w_{0}+w_{1} \sin (z)$ & {$[152]$} & Sine \\
\hline$w(z)=w_{0}+w_{1} \ln (1+z)$ & {$[153]$} & Logarithmic \\
\hline$w(z)=w_{0}+w_{1}\left(\frac{\ln (2+z)}{1+z}-\ln 2\right)$ & {$[154]$} & MZ Model 1 \\
\hline$w(z)=w_{0}+w_{1}\left(\frac{\sin (1+z)}{1+z}-\sin 1\right)$ & {$[154]$} & MZ Model 2 \\
\hline$w(z)=w_{0}+w_{1}\left(\frac{z}{1+z}\right)^{n}$ & {$[155]$} & nCPL \\
\hline$w(z)=w_{0}+w_{1} \frac{z}{(1+z)^{n}}$ & {$[155]$} & nJBP \\
\hline$w(z)=w_{0}+w_{1} \ln \left(1+\frac{z}{1+z}\right)$ & {$[156]$} & Modified Logarithmic \\
\hline
\end{tabular}

$w_{0}, w_{1}$ appearing in the above expressions are constants.

\section{References}

[1] A. Sandage, Physics Today 34 (1970). 
[2] S. Perlmutter et al., Astrophy. J. 517565 (1999).

[3] A. G. Reiss et al., Astron. J. 116, 1009 (1998).

[4] A. G. Riess et al., Astrophy. J. 53662 (2000).

[5] A. G. Riess et al., Astrophy. J. 65998 (2007).

[6] D. H. Weinberg et al., Phys. Rept. 53087 (2013).

[7] P. Astier et al., Astron. \& Astrophy. 44731 (2006).

[8] Amanullah, et al., Astrophy. J. (2010).

[9] D. Rubin et al., Astrophy. J. (2013).

[10] A. H. Jaffe et al., Phys. Rev. Lett. 863475 (2001).

[11] D. N. Spergel et al., Astrophys. J. Suppl. 170377 (2007).

[12] J. R. Bond et al. Mon. Not. R. Astron. Soc. 291 L33 (1997).

[13] Y. Wang and P. Mukherjee, Astrophy. J. 6501 (2006).

[14] U. Seljak et al., Phys. Rev. D 69103501 (2004).

[15] J. K. Adelman-McCarthy et al., Astrophy. J. Suppl. 16238 (2005).

[16] R. G. Vishwakarma, arXiv: 1605.09236v1 (2016).

[17] R. G. Vishwakarma, Int. J. Geom. Meth. Mod. Phys. 121550116 (2015).

[18] R. G. Vishwakarma, Frontiers of Physics 9(1) 98 (2014).

[19] R. G. Vishwakarma, Res. Astron. Astrophys. 131409 (2013).

[20] V. Sahni, Int. J. Mod. Phys. D 9373 (2000).

[21] R. G. Vishwakarma, Mon. Not. R. Astron. Soc. 331776 (2002).

[22] P. J. E. Peebles and B. Ratra, Rev. Mod. Phys. 75559 (2003).

[23] S. Weinberg, Rev. Mod. Phys. 611 (1989).

[24] B. Ratra and P. J. E. Peebles, Phys. Rev. D 37, 3406 (1988).

[25] R. R. Caldwell, R. Dave and P. J. Steinhardt, Phys. Rev. Lett. 801582 (1998).

[26] V. Sahni, M. Sami and T. Souradeep, Phys. Rev. D 65023518 (2002).

[27] M. Sami and T. Padmanabhan, Phys. Rev. D 67083509 (2003).

[28] Armendariz-Picon, T. Damour and V. Mukhanov, Phys. Lett. B 458209 (1999). 
[29] T. Chiba, T. Okabe and M. Yamaguchi, Phys. Rev. D 62023511 (2000).

[30] L. A. Boyle, R. R. Caldwell and M. Kamionkowski, Phys. Lett. B 54517 (2002).

[31] A. Sen, J. High Energy Phys. 0207065 (2002).

[32] T. Padmanabhan, Phys. Rev. D 66021301 (2002).

[33] B. Feng, X. L. Wang and X. M. Zhang, Phys. Lett. B 60735 (2005).

[34] Z. K. Guo, Y. S. Pio, Y. Z. Zhang and X. M. Zhang, Phys. Lett. B 608177 (2005).

[35] M. R. Setare, J. Sadeghi and A. R. Amani, Phys. Lett. B 660299 (2008).

[36] M. R. Setare, E. N. Saridakis, J. Cosmol. Astropart. Phys. 09026 (2008).

[37] J. Khoury, and A. Weltman, Phys. Rev. Lett. 93171104 (2004).

[38] P. Brax, et al., Phys. Rev. D 70123518 (2004).

[39] Abdussattar and S.R. Prajapati, Int. J. Theor. Phys. 502355 (2011).

[40] L. Parker and A. Raval, Phys. Rev. D 60063512 (1999).

[41] V. Sahni and A. Starobinsky, Int. J. Mod. Phys. D 9373 (2000).

[42] S. Nojiri, S. D. Odintsov, Phys. Lett. B 562147 (2003).

[43] Parampreet Singh, M. Sami, Naresh Dadhich, Phys. Rev. D 68 023522(2003).

[44] M. Sami and A. Toporensky, Mod. Phys. Lett. A 191509 (2004).

[45] A. V. Astashenok et al., Phys. Lett. B 709(4) 396 (2012).

[46] A. Yu. Kamenshchik, U. Moschella and V. Pasquier, Phys. Lett. B 511265 (2001).

[47] V. Gorini et al., AIP Conf. Proc. 751108 (2005).

[48] E. J. Copeland, M. Sami and S. Tsujikawa, Int. J. Mod. Phys. D 151753 (2006).

[49] M. Sami, New Adv. Phys. 1077 (2016).

[50] M. Sami, R. Myrzakulov, arXiv:1309.4188v2 (2013).

[51] Md. Wali Hossain et al., Int. J. Mod. Phys. D 241530014 (2015).

[52] M. Sami, Curr. Sci. 97887 (2009).

[53] M Sami, arXiv:0901.0756v1 (2009).

[54] J. Yoo and Y. Watanabe, Int. J. Mod. Phys. D 211230002 (2012).

[55] M. S. Turner and A. G. Riess, Astrophys. J. 56918 (2002). 
[56] S. Weinberg, Cosmology and Gravitation (John Wiley Sons, New York) (1972).

[57] Eric V. Linder, Phys. Rev. D 73063010 (2006).

[58] Yu. L. Bolotin et al., arXiv:1502.00811 (2015).

[59] Yu. L. Bolotin et al., arXiv:1506.08918 (2015).

[60] S. Nojiri, S. D. Odintsov and V. K. Oikonomou, Phys. Lett. B 747310 (2015).

[61] M. S. Berman, Nuovo Cimento 74(2) (1983).

[62] N Banerjee, S. Das and K. Ganguly, Pramana 74(3) 481 (2010).

[63] J. P. Singh, Astrophys. Space Sci. 318103 (2008).

[64] S. K. J. Pacif and B. Mishra, Res. Astron. Astrophys. 15(12) 2141 (2015).

[65] S. K. J. Pacif and B. Mishra, Astrophys. Space Sci. 36048 (2015).

[66] F. Cannata and A. Y. Kamenshchik, Int. J. Mod. Phys. D 20121 (2010).

[67] S. Noniri and S. D. Odintsov, Gen. Relativ. Grav. 38(8) 1285 (2006).

[68] Paul H. Frampton et al., Phys. Lett. B 708204 (2012).

[69] S. Nojiri et al., J. Cosmol. Astropart. Phys. 1509044 (2015).

[70] K. Bamba et al., Phys. Lett. B 732349 (2014).

[71] D. Lohiya and M. Sethi, Class. Quantum Grav. 161545 (1999).

[72] M. S. Berman and F. M. Gomide, Gen Relativ. Grav. 20191 (1998).

[73] A-M. M. Abdel Rahman, Phys. Rev. D 45(10) 3497 (1992).

[74] Abdussattar and S. R. Prajapati, Astrophys. Space Sci. 331657 (2011).

[75] O. Akarsu and T. Dereli, Int. J. Theoret. Phys. 51612 (2012).

[76] Ozgur Akarsu et al., J. Cosmol. Astropart. Phys. 0122 (2014).

[77] B. Mishra and S. K. Tripathy, Modern Physics Letters A 30(36) 1550175 (2015).

[78] J. M. Overduin and F. I. Cooperstock, Phys. Rev. D 58043506 (1998).

[79] R. G. Vishwakarma, Mon. Not. R. Astron. Soc. 345545 (2003).

[80] J. Simon et al., Phys. Rev. D 71123001 (2005).

[81] M. Moresco et al., J. Cosmol. Astropart. Phys. 1208006 (2012).

[82] J. A. Cuesta et al., Mon. Not. R. Astron. Soc. 4571770 (2016). 
[83] C. Blake et al., Mon. Not. R. Astron. Soc. 425405 (2012).

[84] D. Stern et al., J. Cosmol. Astropart. Phys. 1002008 (2010).

[85] M. Moresco et al., J. Cosmol. Astropart. Phys. 1605014 (2016).

[86] T. Delubac et al., Astron. \& Astrophys. 574 A59 (2015).

[87] A. Font-Ribera et al., J. Cosmol. Astropart. Phys. 1405027 (2014).

[88] A. Batra, M. Sethi and D. Lohiya, Phys. Rev. D 60108301 (1999).

[89] G. F. R. Ellis and M. S. Madsen, Class. Quantum Grav. 8667 (1991).

[90] S. D. Odintsov and V. K. Oikonomou, Phys.Rev. D 92(2) 024016 (2015).

[91] S. Nojiri, S. D. Odintsov and S. Tsujikawa, Phys. Rev. D 71063004 (2005).

[92] M. Ozer and M.O. Taha, Phys. Lett. B 171363 (1986).

[93] M. Ozer and M.O. Taha, Nucl. Phys. B 287776 (1987).

[94] A-M. M. Abdel-Rahman, Gen. Relativ. Gravit. 22655 (1990).

[95] S. K. J. Pacif and Abdussattar, Eur. Phys. J. Plus 129244 (2014).

[96] S. K. J. Pacif and Abdussattar, Proceedings of 3rd International Conference on "Innovative Approach in Applied Physical, Mathematical/Statistical, Chemical Sciences and Emerging Energy Technology for Sustainable Development", New Delhi (2014).

[97] J. N. Islam, An Introduction to Mathematical Cosmology (Cambridge University Press, UK) (1992).

[98] Abdussattar and S. R. Prajapati, Chin. Phys. Lett. Vol. 28(2) 029803 (2011).

[99] Abdussattar and R. G. Vishwakarma, Pramana 4741 (1996).

[100] R. G. Vishwakarma, Gen. Relativ. Grav. 33(11) 1973 (2001).

[101] R. G. Vishwakarma, Abdussattar and A. Beesham, Phys. Rev. D 60063507 (1999).

[102] Abdussattar and R. G. Vishwakarma, Aust. J. Phys. 50893 (1997).

[103] T. S. Olson and T. F. Jordan, Phys. Rev. D 353258 (1987).

[104] D. Pavon, Phys. Rev. D 43375 (1991).

[105] R. G. Vishwakarma, Class. Quantum Grav. 18, 1159 (2001).

[106] S. Ray et al., Int. J. Theor. Phys. 48(9) 2499 (2009).

[107] Arbab I Arbab, Gen. Relativ. Grav. 2961 (1997). 
[108] Arbab I Arbab, Chin. Phys. Lett. 254497 (2008).

[109] S. G. Rajeev, Phys. Lett. B 125144 (1983).

[110] A. D. Linde, JETP Lett. 19, 183 (1974).

[111] A. Beesham, Phys. Rev. D 483539 (1993).

[112] J. C. Carvalho, J A S Lima and I Waga, Phys. Rev. D 462404 (1992).

[113] Arbab I Arbab and A.-M. M. Abdel-Rahman, Phys. Rev. D 50(12) 7725 (1995).

[114] A. S. Al-Rawaf, Mod. Phys. Lett. A 13429 (1998).

[115] Arbab I Arbab, Grav. Cosmol. 8227 (2002).

[116] J. W. Moffat, Los Alamos report astro-ph/9608202 (1996).

[117] C. Ekart, Phys. Rev. 58919 (1940).

[118] O. Gron, Astrophys. Space Sci. 17391 (1990).

[119] S. Nojiri and S. D. Odintsov, Phys. Rev. D 70, 103522 (2004).

[120] K. Karami, S. Ghaari, and J. Fehri, Eur. Phys. J. C 64(1) 85 (2009).

[121] Pierre-Henri Chavanis, Eur. Phys. J. Plus 12938 (2014).

[122] Pierre-Henri Chavanis, arXiv:1309.5784v2 (2015).

[123] M. C. Bento, O. Bertolami and A. A. Sen, Phys. Rev. D 66043507 (2002).

[124] V. Gorini, A. Yu. Kamenshchik and U. Moschella, Phys. Rev. D 67063509 (2003).

[125] H. B. Benaoum, arxiv:hep-th/0205140v1 (2002)

[126] U. Debnath, A. Banerjee, and S. Chakraborty, Class. Quantum Grav. 215609 (2004).

[127] U. Debnath, Astrophys. Space Sci. 312295 (2007).

[128] W. Chakraborty, U. Debnath, Gravit. and Cosmol. 16223 (2010).

[129] M. S. Berman, Phys. Rev. D 431075 (1991).

[130] N. Banerjee and S. Das, Gen. Relativ. Grav. 371695 (2005).

[131] A. G. Riess et al., Astrophy. J. 607665 (2004).

[132] J. V. Cunha and J. A. S. Lima, Mon. Not. R. Astron. Soc. 390210 (2008).

[133] J. V. Cunha, Phys. Rev. D 79047301 (2009).

[134] R. Nair et al., J. Cosmol. Astropart. Phys. 01018 (2012). 
[135] B. Santos, J. C. Carvalho, J. S. Alcaniz, Astropart. Phys. 3517 (2011).

[136] L. Xu and H. Liu, Mod. Phys. Lett. A 231939 (2008).

[137] Abdulla Al Mamon and Sudipta Das, arXiv:1507.00531v1 (2015).

[138] Y. G. Gong and A. Wang, Phys. Rev. D 73083506 (2006).

[139] J. Lu, L. Xu and M. Liu, Phys. Lett. B 699246 (2011).

[140] Y. G. Gong and A. Wang, Phys. Rev. D 75043520 (2007).

[141] S. del Campo et al., Phys. Rev. D 86083509 (2012).

[142] D. Pavon et al., Proc. MG13 Meeting Gen. Relativ., Stockholm Univ., Sweden (2012). arXiv:1212.6874v1.

[143] E. E. O. Ishida et al., Astroparticle Phys. 287 (2007).

[144] D. Huterer, M. S. Turner, Phys. Rev. D 64123527 (2001).

[145] J. Weller and A. Albrecht, Phys. Rev. D 65103512 (2002).

[146] H. K. Jassal, J. S. Bagla, and T. Padmanabhan, Mon. Not. R. Astron. Soc. Letters 356(1) L11 (2005).

[147] M. Chevallier and D. Polarski, Int. J. Mod. Phys. D 10213 (2001).

[148] E. V. Linder, Phys. Rev. Lett. 90091301 (2003).

[149] G. Pantazis, S. Nesseris and L. Perivolaropoulos, Phys. Rev. D 93103503 (2016).

[150] E. M. Barboza, Jr. and J. S. Alcaniz, J. Cosmol. Astropart. Phys. 02042 (2012).

[151] C. -J. Feng et al., J. Cosmol. Astropart. Phys. 09023 (2012).

[152] R. Lazkoz, V. Salzano, and I. Sendra, Phys. Lett. B 694198 (2010).

[153] G. Efstathiou, Mon. Not. R. Astron. Soc. 310842 (1999).

[154] J. -Z. Ma and X. Zhang, Phys. Lett. B 699233 (2011).

[155] Dao-Jun Liu et al., Mon. Not. R. Astron. Soc. 388275 (2008).

[156] L. Feng and T. Lu, J. Cosmol. Astropart. Phys. 11034 (2011). 